Atsushi Saito - Toru Furukawa - Shinichi Fukushige

Shohei Koyama • Masato Hoshi • Yutaka Hayashi

Akira Horii

\title{
p24/ING1-ALT1 and p47/ING1-ALT2, distinct alternative transcripts of $\mathrm{p33/ING1}$
}

Received: November 12, 1999 / Accepted: December 20, 1999

\begin{abstract}
ING1s (the growth inhibitor ING1 and candidate tumor suppressor ING1) are key players in the suppressive pathways for human tumorigenesis. We analyzed their complete transcripts, primary structures, and expression. The results led us to discover two novel and related alternatively spliced transcripts encoding p24/ING1-ALT1 and $\mathrm{p} 47 / \mathrm{ING} 1-\mathrm{ALT} 2$. They share C-terminal residues with the candidate tumor suppressors $\mathrm{p} 33 / \mathrm{ING} 1$. The candidate tumor suppressors p33/ING1 and p24/ING1-ALT1 were coexpressed in a variety of fetal and adult human tissues, but p47/ING1-ALT2 was minimally expressed.
\end{abstract}

Key words ING1 · p33 · Alternative splicing · p24/ING1ALT1 · p47/ING1-ALT2 - Chromosome band 13q34

\section{Introduction}

p33/ING1 has been cloned and mapped to $13 \mathrm{q} 34$ (Garkavtsev et al. 1996; Garkavtsev et al. 1997), a region frequently rearranged in gastric cancers and head and neck squamous cell carcinomas (Motomura et al. 1988; Maestro et al. 1996). The p33/ING1 protein cooperates with p53 in the negative regulatory pathway of cell growth by modulating p53-dependent transcriptional activation (Garkavtsev et al. 1998). These findings suggest that p33/ ING1 is one of the key players in the suppressive pathway for tumorigenesis.

Two kinds of transcripts have been reported to encode

A. Saito $\cdot$ T. Furukawa $\cdot$ S. Fukushige $\cdot$ S. Koyama $\cdot$ M. Hoshi $\cdot$

A. Horii $(\bowtie)$

Department of Molecular Pathology, Tohoku University School of Medicine, 2-1 Seiryo-machi, Aoba-ku, Sendai, Miyagi 980-8575,

Japan

Tel. +81-22-717-8042; Fax +81-22-717-8047

e-mail: horii@mail.cc.tohoku.ac.jp

Y. Hayashi

Department of Pediatric Oncology, Tohoku University School of

Medicine, Sendai, Japan the p33/ING1 proteins. One was identified as the growth inhibitor ING1 (p33/ING1-GI) (see GenBank accession number AF001954) (Benson et al. 1999) and the other as the candidate tumor suppressor ING1 (p33/ING1-TS) (see GenBank accession number AF044076). These proteins harbor identical C-terminal residues but different N-terminal residues. Because there is no information about their primary structures or physiological expression, their identities and relationships are unclear.

\section{Methods and results}

We initially performed reverse transcription-polymerase chain reaction (RT-PCR), using sense primers specific for p33/ING1-TS (TSNF, 5'-AAAGGATCCACCATGTTGAGTCCTGCCAAC-3') and p33/ING1-GI (GIF, 5'TTTGGATCCAATATGCCGTTGTGCACGGC-3') and a common antisense primer ( $\mathrm{R}, 5^{\prime}$-TTTCTCGAGTTGCACCTCAACAAAGGCAGC-3'), but we obtained only a specific PCR product for p33/ING1-TS. To identify the p33/ING1-GI transcript, we performed 5'RACE (rapid amplification of cDNA ends), using total RNA of adult cerebrum and primers UR (for reverse-transcription, 5'ACAGACAGTACGTGGGTTCGTTG-3') and C12 (for PCR, 5'-TTGGAATTCGAAACTGTCTCCGAGCCGG$3^{\prime}$ ), according to methods described previously (Horii et al. 1993). After the sequencing analysis, we found that the RACE clones harbored nucleotide sequences similar to but distinct from that of the p33/ING1-GI. The great majority of the clones harbored the same sequence. The size of this transcript was estimated as 2286 nucleotides, consisting of potentially encoding identical C-terminal but different $\mathrm{N}$ terminal sequences from p33/ING1-GI; the 292 nucleotides in the $5^{\prime}$ end were different from $p 33 / I N G 1-G I$. This transcript encoded a polypeptide of 210 residues with an estimated molecular weight of $24 \mathrm{kDa}$; we named it p24/ ING1-ALT as the alternative transcript of ING1 (see below). In the $5^{\prime}$ portion upstream of the predicted initiating ATG codon of the $p 24 / I N G 1-A L T$ transcript, there was a 
region potentially encoding continuous open reading frame (ORF).

We then performed a second 5' RACE analysis, using primers C18 (5'-AGCAGCGAGAAGAACCAATC-3') and $\mathrm{C} 22$ (5'-CCGGAATTCGACCCGAAGACGTTCAAATC-3') for reverse-transcription and PCR, respectively, and we got two bands; one was about $150 \mathrm{bp}$, which was compatible with the p24/ING1-ALT transcript, and the other was about $700 \mathrm{bp}$. We cloned the $700-\mathrm{bp}$ product and obtained 928 clones. We determined the nucleotide sequences in three clones and found that all of them harbored the same sequence with different-sized poly(A) stretches; two had 17 and one had 19 adenine residues. The transcript spanned 2871 nucleotides, including the complete transcript of $p 24 / I N G 1-A L T$, with an additional 585 nucleotides in the $5^{\prime}$ end. This transcript encoded a polypeptide consisting of 422 amino-acid residues with an estimated molecular weight of $47 \mathrm{kDa}$; we named it $p 47 / I N G 1-A L T 2$ as the alternative transcript-2 of ING1 (also see below), and we renamed $p 24 / I N G 1-A L T$ to $p 24 / I N G 1-A L T 1$ as the alternative transcript-1 of ING1. We could not obtain a transcript identical to $p 33 / I N G 1-G I$. For $p 33 / I N G 1-T S$, we also performed $5^{\prime}$ RACE with a primer set of UR and TS5'R (5'-CAGGAATTCCTCCACATAGTTCACCAG$3^{\prime}$ ) for reverse-transcription and PCR, respectively, and identified a 2431-nucleotide transcript, including the published nucleotide sequence, with newly identified additional nucleotides corresponding to the $5^{\prime}$ untranslated region $\left(5^{\prime}\right.$
UTR). The transcripts of $p 24 / I N G 1-A L T 1, p 47 / I N G 1-$ $A L T 2$, and $p 33 / I N G 1-T S$ shared the same $3^{\prime}$ portion for 1845 nucleotides; the predicted ORF for each transcript led to an identical frame in the common C-terminal region. Hence, the predicted protein products of these three transcripts would share the same C-terminal polypeptides. The entire ORF of $p 24 / I N G 1-A L T 1$ was included in this common C-terminal region. Complete nucleotide sequences for p33/ING1-TS, p24/ING1-ALT1, and p47/ING1-ALT2 were deposited in the DDBJ/EMBL/GenBank databases under the accession numbers AB024401, AB031269, and AB024402, respectively.

To determine primary structures, we screened a human BAC library (Research Genetics, Huntsville, AL, USA) that harbored $p 24 / I N G 1-A L T 1$ and $p 47 / I N G 1-A L T 2$ by a PCR-based method (Kimura et al. 1998), with the primer set 5'-ACAACGAGAACCGTGAGAACGC-3' and 5'TTGCACCTCAACAAAGGCAGC-3'. BAC b585K18 was isolated, and nucleotide sequencing was performed. A comparison of nucleotide sequences between BAC and cDNAs led us to find that this BAC clone harbored all the exons for the two types of transcripts. The nucleotide sequences of the exon-intron boundaries are summarized in Table 1. Moreover, exonic sequences encoding $p 33 / I N G 1$ $T S$ were also included in this BAC clone. The second exon was identical for all the genes, but the first exons were distinct for each gene (Fig. 1). For the p33/ING1-TS gene, exon 1 containing the $5^{\prime}$ UTR and the $5^{\prime}$ portion of the

Table 1. Nucleotide sequences of the exon-intron boundaries

\begin{tabular}{lll}
\hline & Splice donor site & Splice acceptor site \\
\cline { 2 - 3 } & Exon 1/intron 1 & Intron 1/exon 2 \\
\hline p33/ING1-TS & AAATACCAAGgtacggccgggtgatggatg & ctgtccttcttgcccccagAGATCCTGAA \\
ctglcttcttgccccagAGATCCTGAA
\end{tabular}

Nucleotide sequences in upper- and lowercase indicate exonic and intronic sequences, respectively

Fig. 1. Primary structures of $p 33 /$ ING1-TS, p24/ING1-ALT1, and p47/ING1-ALT2. Boxes indicate exons. Dashed lines around boxes indicate open reading frames. Length of exons is indicated $b e$ low the boxes. Start and stop codons ATG and TAG are indicated. Arrows indicate relative positions of primers used for reverse transcriptionpolymerase chain reaction (RTPCR) and 5' RACE. (rapid amplification of cDNA ends) Sizes of introns were not determined

\section{Exon 1}

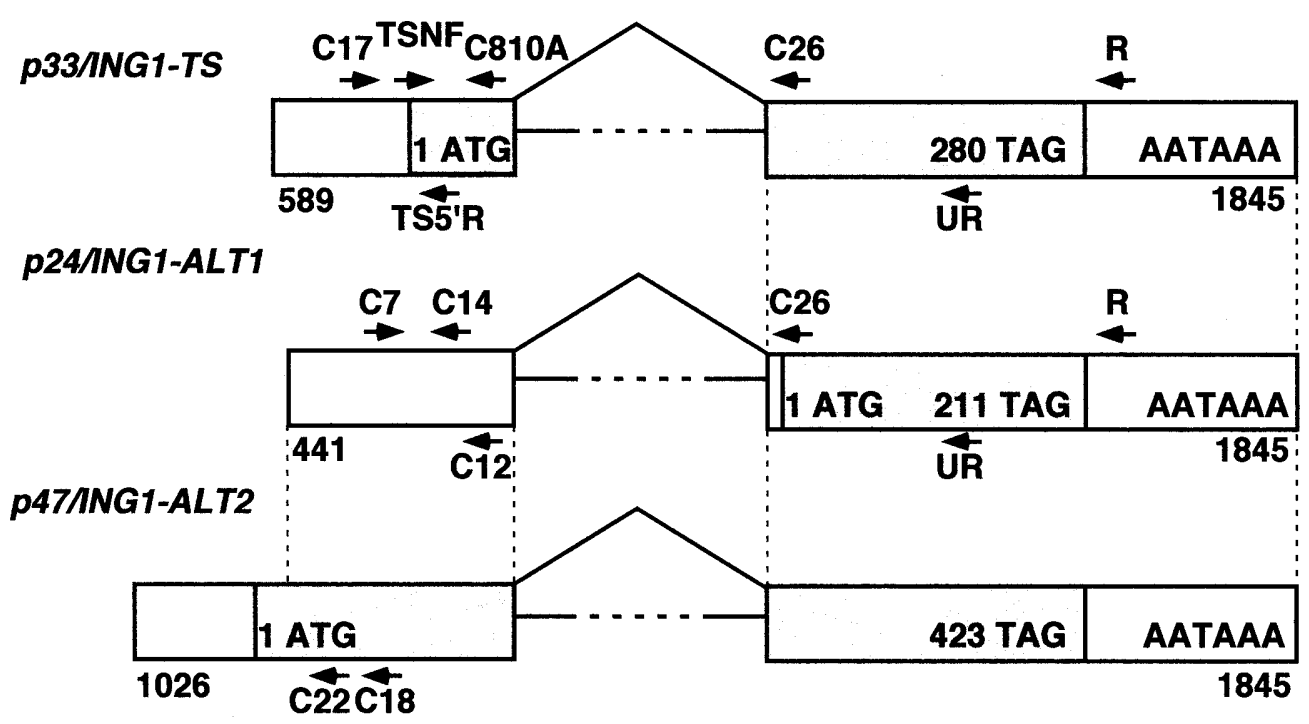


coding region was also distinct from those of the p24/ING1$A L T 1$ and $p 47 / I N G 1-A L T 2$ genes; however, exon 2, including the polyadenylation signal, was shared with these transcripts. For $p 24 / I N G 1-A L T 1$, exon 1 corresponded to the $5^{\prime}$ UTR, while exon 2 contained the entire coding region. For $p 47 / I N G 1-A L T 2$, exon 1 consisted of the entire exon 1 of $p 24 / I N G 1-A L T 1$ and an additional continuous 5' portion harboring a predicted initiating ATG codon. Exon 2 of $p 47 / I N G 1-A L T 2$ was identical to that of $p 24 / I N G 1$ $A L T 1$ and $p 33 / I N G 1-T S$. The primary structures, along with the predicted initiating codon of $p 24 / I N G 1-A L T 1$ and p47/ING1-ALT2 are shown in Fig. 1. Lengths of introns were not determined. Nucleotide sequences of exon-intron junctions were deposited to DDBJ/EMBI/GenBank databases under the accession numbers AB024403 through AB024405. The complete nucleotide sequence of the commonly shared exon 2 in the BAC clone revealed some differences from the published sequence of p33/ING1-TS; from GTG to GCG (V123A), from GTT to GCT (V129A), from AAT to AAA (N135K), from GAT to GAG (D137E), from GTA to GCA (V139A), and from TCT to GCT (S142A). We sequenced 50 alleles of 25 healthy Japanese volunteers' genomic DNA to examine the incidence of these alterations and found that they prevailed in all specimens.

As there were some differences in nucleotide sequence between the published p33/ING1-TS and the clones we isolated, and as we could not detect the p33/ING1-GI transcript (see below), we explored the possibility that the $p 33 /$ ING1 genes we detected were transcribed from a locus other than that of the original $p 33 / I N G 1$ transcripts. Fluorescent in situ hybridization (FISH) analysis with the BAC clone b585K18 revealed that this gene mapped to $13 \mathrm{q} 34$ (see Fig. 2), the same locus as the p33/ING1 reported by Garkavtsev et al. (1997).

To study the expressions of $p 33 / I N G 1-T S, p 24 / I N G 1$ $A L T 1$, and $p 47 / I N G 1-A L T 2$, we performed Northern blot analysis and RT-PCR. Human multiple tissue Northern (MTN) blot, human MTN-IV, and human fetal MTN-II (Clontech, Palo Alto, CA, USA) were hybridized with a radiolabelled cDNA fragment corresponding to the commonly shared region encoding the C-terminus. Distinct transcripts, $4.0-\mathrm{kb}, 2.8-\mathrm{kb}$, and $2.4-\mathrm{kb}$ in size, were detected in fetal tissues, but not in adult tissues (Fig. 3A). Hybridization of the blots with a cDNA fragment specific for $p 33 /$ ING1-TS detected only a $2.8-\mathrm{kb}$ transcript (data not shown). Hybridization of the blots with cDNA fragments specific for $p 24 / I N G 1-A L T 1$ or $p 47 / I N G 1-A L T 2$ gave no specific signals (data not shown), suggesting low-level expression of these transcripts. Hence, the origins of the transcripts that were $4.0-\mathrm{kb}$ and $2.4-\mathrm{kb}$ in size were not clear. Multiplex RT-PCR was carried out to analyze the expression of p33/ING1-TS, p24/ING1-ALT1, and p47/ING1$A L T 2$, using total RNAs of various adult and fetal normal tissues, including adult heart, adult lung, adult kidney, adult rectum, adult liver, fetal brain, fetal liver, and fetal intestine, according to a method described previously (Mori et al. 1997). Southern hybridization of the PCR products, using distinct internal radiolabelled oligo-

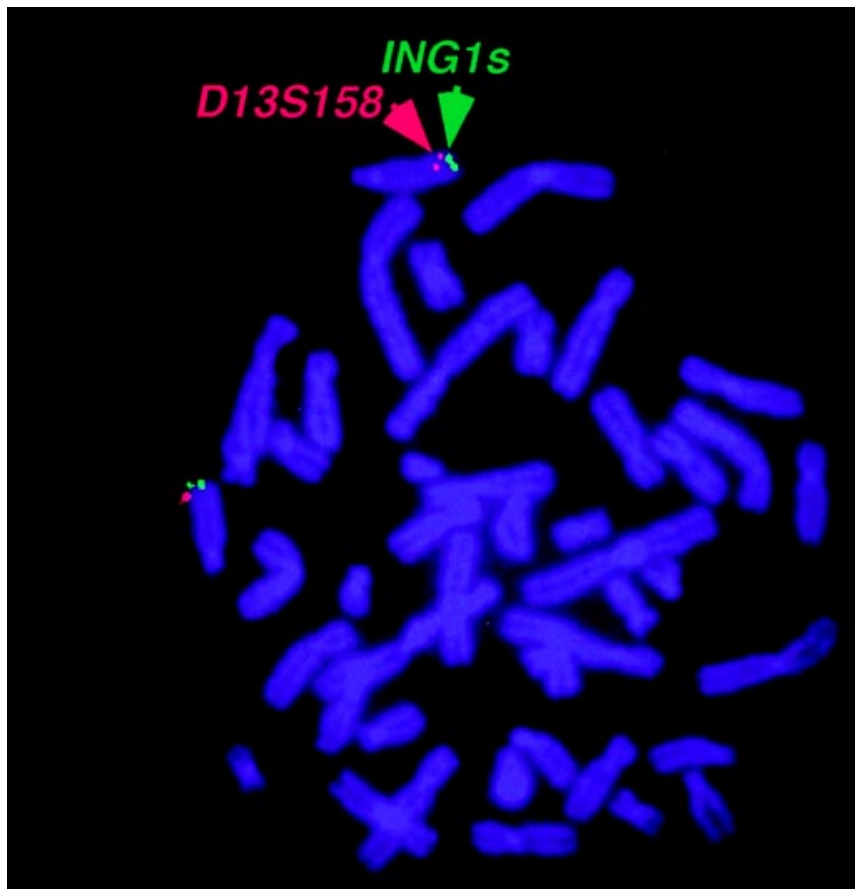

Fig. 2. Two-color fluorescent in situ hybridization (FISH) analysis employing BAC clones b585K18 harboring p24/ING1-ALT1, p47/ ING1-ALT2, and p33/ING1-TS (green) and b535P6 harbouring D13S158 (red) on human lymphocyte metaphase chromosomal spread clearly indicates $I N G 1$ genes localize on $13 \mathrm{q} 34$

nucleotides for those genes, showed expression of $p 33 /$ ING1-TS and p24/ING1-ALT1, but no expression of $p 47 /$ ING1-ALT2 in all tested tissues (Fig. 3B). It was postulated that physiological expression of $p 47 / I N G 1-A L T 2$ may be rare.

\section{Discussion}

We identified two novel alternatively spliced transcripts, p24/ING1-ALT1 and p47/ING1-ALT2, by means of $5^{\prime}$ RACE; these are transcribed from two very closely localized but distinct promoter regions. We detected the expression of p24/ING1-ALT1 in a variety of normal tissues by RT-PCR but could not detect p47/ING1-ALT2. We think that the rare physiological expression of p47/ING1-ALT2 prevented us from finding the transcript by conventional RT-PCR in normal tissues. As described by Helbing et al. (1997) and Garkavtsev et al. (1998), p33/ING1 is induced by stimulation of the induction of apoptosis and cooperates with p53 in cell growth control. We speculate that p24/ ING1-ALT1 and p47/ING1-ALT2 may also be induced and play a role in the apoptotic pathway. Further studies will necessary to clarify the roles of these two products in physiological and pathological conditions. We could not detect the transcript of $p 33 / I N G 1-G I$ in any fetal or adult human tissues by RT-PCR or 5' RACE. This transcript may be very rarely expressed or may be affected by some rearrangement. Our present results will contribute to the effort to clarify the physiological and pathological roles of $p 33 /$ 

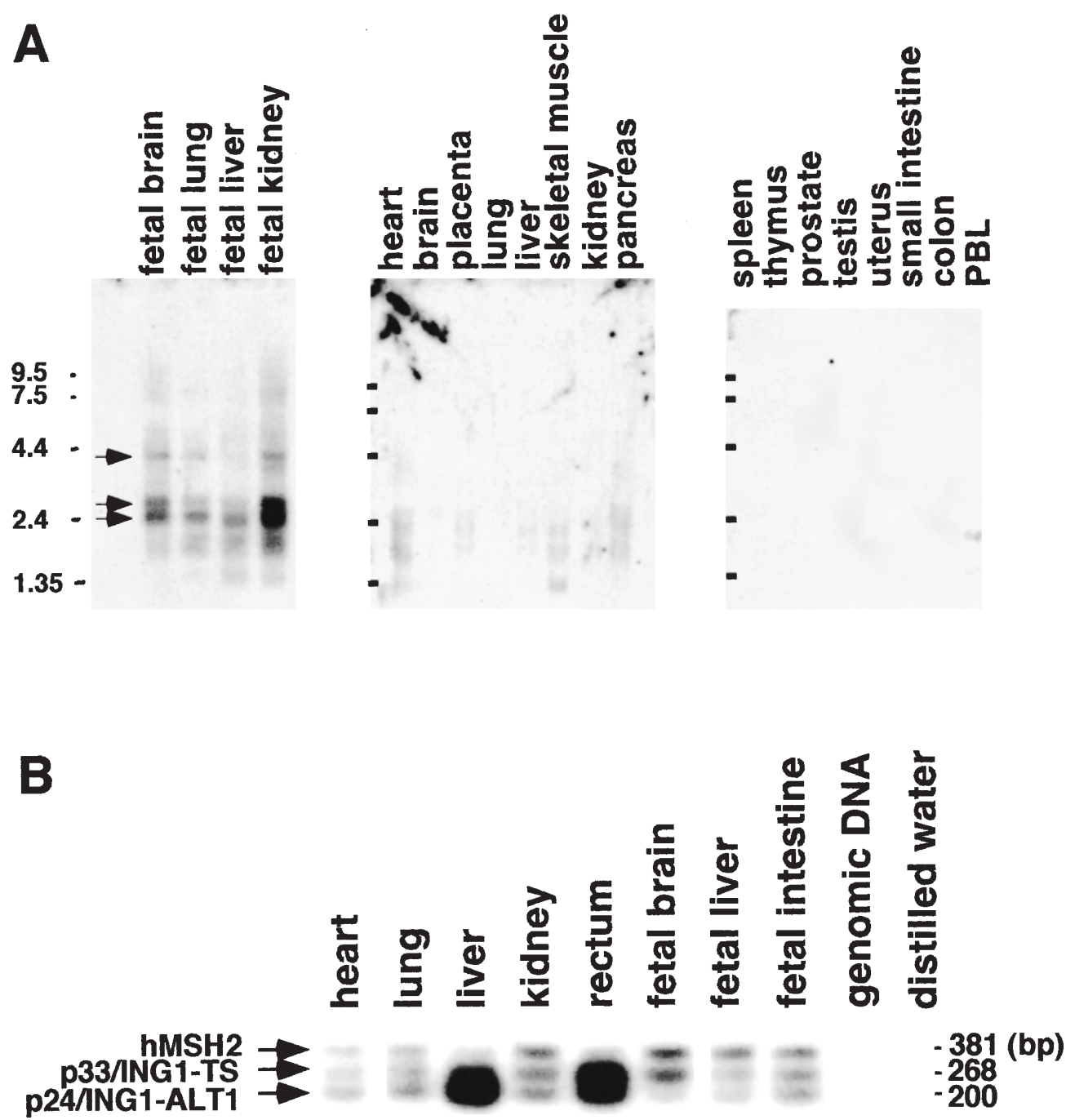

Fig. 3. A Human multiple tissue Northern (MTN) blot, human MTNIV, and human fetal MTN-II (Clontech, Palo Alto, CA, USA) were hybridized with a radiolabelled cDNA fragment corresponding to the commonly shared C-terminal coding region. Distinct transcripts, $4.0-\mathrm{kb}, 2.8-\mathrm{kb}$, and $2.4-\mathrm{kb}$ in size, were detected in fetal tissues but not in adult tissues. B RT-PCR to detect expression of $p 24 / I N G 1-A L T 1$ and $p 33 / I N G 1$ in normal adult and fetal tissues. First-strand cDNA was subjected to a 30-cycle amplification comprising incubations at $94^{\circ} \mathrm{C}$ for $30 \mathrm{~s}, 60^{\circ} \mathrm{C}$ for $30 \mathrm{~s}$, and $72^{\circ} \mathrm{C}$ for $30 \mathrm{~s}$, followed by a final incubation at $72^{\circ} \mathrm{C}$ for $5 \mathrm{~min}$ with primers of $\mathrm{C} 17$ ( $5^{\prime}$-CGAGGGCTTTGCATTTTGCA-3'), C7 (5'-ACTTTCGGGCGCGGATTTAT-3'), and C26 (5'-

ING1-TS, as well as those of p24/ING1-ALT1 and p47/ ING1-ALT2.

Acknowledgments We are grateful to Dr. Barbara Lee Smith Pierce (the Life Science Coordinator for the University of Maryland Asian Division) for editorial work in the preparation of this manuscript. This work was supported by the Ministry of Education, Science, Sports, and Culture of Japan.

\section{References}

Benson DA, Boguski MS, Lipman DJ, Ostell J, Ouellette BF, Rapp BA, Wheeler DL (1999) GenBank. Nucleic Acids Res 27:12-17

Garkavtsev I, Demetrick D, Riabowol K (1997) Cellular localization

CGCTCGTAGCACTCGTCTAG-3') in a GeneAmp PCR system 9600 (Perkin-Elmer Applied Biosystems, Foster City, CA, USA). $h M S H 2$ was co-amplified with primers of 5'-TTGGGAAGAGGAACTTGTACC-3' and 5'-TTCTTTGCTGCTGGTTCCATG-3' as an internal control. PCR products were blotted and hybridized with ${ }^{32} \mathrm{P}$-end-labelled internal oligonucleotides of C810A (5'-ATCAGCGAGACATTTCTCTG-3'), detecting a 268-bp product for $p 33 / I N G 1$ TS; C14 (5'-GGGATCACTGCTACTGCTA-3'), detecting a 200-bp product for $p 24 / I N G 1-A L T 1$; and 5'-ACTGTTAATAATCTACATGTCACA-3' detecting a 381-bp product for $h M S H 2$. Relative positions of primers are shown in Fig. 1. PBL, Peripheral blood leukocytes

and chromosome mapping of a novel candidate tumor suppressor gene (ING1). Cytogenet Cell Genet 76:176-178

Garkavtsev I, Grigorian IA, Ossovskaya VS, Chernov MV, Chumakov PM, Gudkov AV (1998) The candidate tumour suppressor p33 $3^{\mathrm{ING} 1}$ cooperates with p53 in cell growth control. Nature 391:295-298

Garkavtsev I, Kazarov A, Gudkov A, Riabowol K (1996) Suppression of the novel growth inhibitor $\mathrm{p} 33^{\mathrm{ING} 1}$ promotes neoplastic transformation. Nat Genet 14:415-420

Helbing CC, Veillette C, Riabowol K, Johnston RN, Garkavtsev I (1997) A novel candidate tumor suppressor, ING1, is involved in the regulation of apoptosis. Cancer Res 57:1255-1258

Horii A, Nakatsuru S, Ichii S, Nagase H, Nakamura Y (1993) Multiple forms of the APC gene transcripts and their tissue-specific expression. Hum Mol Genet 2:283-287

Kimura M, Furukawa T, Abe T, Yatsuoka T, Youssef EM, Yokoyama T, Ouyang H, Ohnishi Y, Sunamura M, Kobari M, Matsuno S, Horii A (1998) Identification of two common regions of allelic loss in chromosome arm $12 \mathrm{q}$ in human pancreatic cancer. Cancer Res 
58:2456-2460

Maestro R, Piccinin S, Doglioni C, Gasparotto D, Vukosavljevic T, Sulfaro S. Barzan L, Boiocchi M (1996) Chromosome 13q deletion mapping in head and neck squamous cell carcinomas: identification of two distinct regions of preferential loss. Cancer Res 56:1146-1150 Mori Y, Shiwaku H, Fukushige S, Wakatsuki S, Sato M, Nukiwa T,
Horii A (1997) Alternative splicing of $h M S H 2$ in normal human tissues. Hum Genet 99:590-595

Motomura K, Nishisho I, Takai S, Tateishi H, Okazaki M, Yamamoto M, Miki T, Honjo T, Mori T (1988) Loss of alleles at loci on chromosome 13 in human primary gastric cancers. Genomics 2:180-184 